\title{
ARTICLE
}

\section{Optimization by parameters in the iterative methods for solving non-linear equations}

\author{
Tugal Zhanlav ${ }^{1}$ and Khuder Otgondorj ${ }^{1,2 *}$ \\ ${ }^{1}$ Simulation and Computing Department, Institute of Mathematics and Digital Technology, \\ Mongolian Academy of Sciences, Ulaanbaatar, Mongolia \\ ${ }^{2}$ School of Applied Sciences, Mongolian University of Science and Technology,
} Ulaanbaatar, Mongolia

ARTICLE INFO: Received: 23 Apr, 2021; Accepted: 13 Aug, 2021

\begin{abstract}
In this paper, we used the necessary optimality condition for parameters in a two-point iterations for solving nonlinear equations. Optimal values of these parameters fully coincide with those obtained in [6] and allow us to increase the convergence order of these iterative methods. Numerical experiments and the comparison of existing robust methods are included to confirm the theoretical results and high computational efficiency. In particular, we considered a variety of real life problems from different disciplines, e.g., Kepler's equation of motion, Planck's radiation law problem, in order to check the applicability and effectiveness of our proposed methods.
\end{abstract}

Keywords: Iterative methods; Optimal values of parameters; Iterations with memory;

\section{INTRODUCTION}

Many iterative methods for solving nonlinear equations often include non-zero free parameters. Their suitable choices allow to increase the convergence order of methods. There are some choices of parameters based on the error analysis, see for example $[1,2,3,4,5,8,9]$ and references therein. Recently, researchers have also proposed several biparametric two-step simple methods with and without memory $[1,3,4,6]$. On the other hand, optimization by parameters is one of the powerful techniques in science and engineering practice. The main goal of this paper is to find the optimal choices of parameters $\bar{\tau}_{n}$ and, $\gamma$ in the two points iterative methods. We obtained analytic formulae for $\lambda$ and $\gamma$ without symbolic computation technique.

The paper is organized as follows. In section 2 , we developed necessary optimality condition for parameters in the two-point method. In the last section, we present the results of numerical experiments that confirm the theoretical conclusion about the convergence order and made a comparison with well-known methods of the same order of convergence.

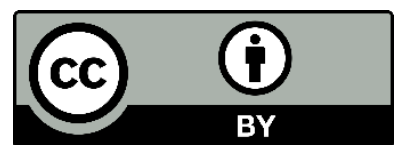

The Author(s). 2021 Open access This article is distributed under the terms of the Creative Commons Attribution 4.0 International License (https://creativecommons.org/licenses/by/4.0/), which permits unrestricted use, distribution, and reproduction in any medium, provided you give appropriate credit to the original author(s) and the source, provide a link to the Creative Commons license, and indicate if changes were made. 
In numerical analysis and engineering applications, it is often required to solve a nonlinear equation $f(x)=0$, where $f(x): D \subset R \rightarrow R$ is a scalar function defined on an open interval $D$. Assume that $f$ is sufficiently smooth and has a simple zero $x^{*} \in$ $D$ and $f^{\prime}$ is nonsingular in $D$.

In this paper, we consider optimization by parameters in the two-point iterative methods $[6,7]$

$$
\begin{aligned}
& y_{n}=x_{n}-\frac{f\left(x_{n}\right)}{\phi_{n}+\lambda f\left(w_{n}\right)}, w_{n}=x_{n}+\gamma f\left(x_{n}\right), \\
& x_{n+1}=y_{n}-\bar{\tau}_{n} \frac{f\left(y_{n}\right)}{\phi_{n}+\lambda f\left(w_{n}\right)}, \phi_{n}=\frac{f\left(w_{n}\right)-f\left(x_{n}\right)}{\gamma f_{n}},
\end{aligned}
$$

where $\gamma, \lambda$ are non-zero parameters. It is easy to show that the minimization problem

$$
\min _{\bar{\tau}_{n}, \lambda, \gamma} f\left(x_{n+1}\right)^{2}
$$

is equivalent to

$$
f\left(x_{n+1}\right)=0 .
$$

\section{Necessary optimality of condition for parameters in the two-point iterations}

The Taylor expansion of $f\left(x_{n+1}\right)$ at the point $y_{n}$ gives

$$
f\left(x_{n+1}\right)=\left(1-\bar{\tau}_{n} \frac{f^{\prime}\left(y_{n}\right)}{\phi_{n}+\lambda f\left(w_{n}\right)}\right) f\left(y_{n}\right)+O\left(f\left(y_{n}\right)^{2}\right) .
$$

From (4) it clear that the problem (3) leads to minimization of $f\left(y_{n}\right)$. If we choose $\bar{\tau}_{n}$ such that

$$
\bar{\tau}_{n}=\frac{\phi_{n}+\lambda f\left(w_{n}\right)}{f^{\prime}\left(y_{n}\right)}
$$

Then we have

$$
f\left(x_{n+1}\right)=O\left(f\left(y_{n}\right)^{2}\right) .
$$

We find stationary point of $f\left(y_{n}(\lambda, \gamma)\right)$ solving the system

$$
\begin{aligned}
& \frac{\partial y_{n}}{\partial \lambda}=f^{\prime}\left(y_{n}\right) \frac{\partial y_{n}}{\partial \lambda}=0, \\
& \frac{\partial y_{n}}{\partial \gamma}=f^{\prime}\left(y_{n}\right) \frac{\partial y_{n}}{\partial \gamma}=0,
\end{aligned}
$$

Since $\mathrm{f}^{\prime}(\mathrm{x}) \neq 0$ in the domain $D$ containing the zero of the equation (3) the system (7) equivalent to the system:

$$
\frac{\partial y_{n}}{\partial \lambda}=0, \frac{\partial y_{n}}{\partial \gamma}=0
$$

From the first equation in (1) we find that

$$
\left\{\begin{array}{l}
\frac{\partial y_{n}}{\partial \lambda}=\frac{f\left(x_{n}\right)}{\left(\phi_{n}+\lambda f\left(w_{n}\right)\right)^{2}} f\left(w_{n}\right), \\
\frac{\partial y_{n}}{\partial \gamma}=\frac{f\left(x_{n}\right)}{\left(\phi_{n}+\lambda f\left(w_{n}\right)\right)^{2}}\left(\frac{\partial \phi_{n}}{\partial \gamma}+\lambda \frac{\partial f\left(w_{n}\right)}{\partial \gamma}\right)
\end{array}\right.
$$


Substituting (9) into (8) we get

$$
\left\{\begin{array}{l}
f\left(w_{n}\right)=0, \\
\frac{\partial \phi_{n}}{\partial \gamma}+\lambda \frac{\partial f\left(w_{n}\right)}{\partial \gamma}=0 .
\end{array}\right.
$$

On the other hand, by definition of functions $w_{n}$ and $\phi_{n}$ we have

$$
f\left(w_{n}\right)=f\left(x_{n}\right)\left(1+f^{\prime}\left(x_{n}\right) \gamma+\frac{f^{\prime \prime}\left(x_{n}\right)}{2} \gamma^{2} f\left(x_{n}\right)+\cdots\right),
$$

and

$$
\begin{aligned}
& \frac{\partial \phi_{n}}{\partial \gamma}+\lambda \frac{\partial f\left(w_{n}\right)}{\partial \gamma}=f\left(x_{n}\right)\left(\frac{f^{\prime \prime}\left(x_{n}\right)}{2}\right. \\
& \left.+\lambda f^{\prime}\left(x_{n}\right)+\gamma f\left(x_{n}\right)\left(\frac{2 f^{\prime \prime \prime}\left(x_{n}\right)}{3 !}+\lambda f^{\prime \prime}\left(x_{n}\right)\right)+\cdots\right),
\end{aligned}
$$

From (11) and (12) clear that the parameters $\gamma$ and $\lambda$ are determined, so that the system of equations (10) holds at least $f\left(y_{n}(\lambda, \gamma)\right)$ with accuracy $O\left(f\left(x_{n}\right)^{2}\right)$. That is

$$
\gamma=\gamma_{n}=-\frac{1}{f^{\prime}\left(x_{n}\right)}, \lambda=\lambda_{n}=-\frac{f^{\prime \prime}\left(x_{n}\right)}{2 f^{\prime}\left(x_{n}\right)}
$$

Thus, we find the extremum point (13) of function $f\left(y_{n}(\lambda, \gamma)\right)$ with accuracy $O\left(f\left(x_{n}\right)^{3}\right)$, because of (9), (11), (12).

The parameters given by the formulae (13) are naturally said to be optimal in the sense of necessary optimality condition (8). So, We can find approximations of $\gamma_{n}, \lambda_{n}$. They can be found using the information available from the current and previous iteration steps. The methods containig such parameters are called methods with memory.

Note that (13) fully coincides with those obtained in [3] and

$$
f\left(y_{n}\right)=O\left(f\left(x_{n}\right)^{4}\right),
$$

under (13). Now we shall find iteration parameter $\bar{\tau}_{n}$ with some accuracy. To this end, we approximate $f^{\prime}\left(y_{n}\right)$ in (5) as:

$$
f^{\prime}\left(y_{n}\right)=\frac{f\left(y_{n}+\Delta_{n}\right)-f\left(y_{n}-\Delta_{n}\right)}{2 \Delta_{n}}+O\left(\Delta_{n}^{2}\right),
$$

where

$$
\Delta_{n}=f\left(x_{n}\right)^{p}, p=1,2 .
$$

As a result, we have

$$
\bar{\tau}_{n}=\frac{2\left(\phi_{n}+\lambda f\left(x_{n}\right)\right) \Delta_{n}}{f\left(y_{n}+\Delta_{n}\right)-f\left(y_{n}-\Delta_{n}\right)} .
$$

From (4) and (15) we conclude that

$$
f\left(x_{n+1}\right)=O\left(f\left(x_{n}\right)^{4+2 p}\right), p=1,2,
$$

under choices (13), (17).

Thus, we can formulate the above considered results as: 
Theorem 1. Assume that $f\left(x_{n}\right)$ sufficiently smooth in $D$ containing the localized zero of the equation (3) and the initial approximation $x_{0}$ is close enough to $x^{*}$. Then the two point iterative method (1) has $4+2 p$, $p=1,2$ order of convergence under choices (13) and (17).

Remarks. Obviously, the optimal choices (13) also hold true for three-point iterative methods, the first two steps of which are the same as (1). Note that the stationary point of view is also applicable for solving system of nonlinear equations.

Based on the choices (13), (17) one can construct new $4+2 p$ order convergence iterations with memory:

$$
\begin{aligned}
& x_{0}, \lambda_{0}, \gamma_{0} \text { are given, then } w_{0}=x_{0}+\gamma_{0} f\left(x_{0}\right), \\
& \gamma_{n}=-\frac{2 f\left(x_{n}\right)}{f\left(x_{n}+f\left(x_{n}\right)\right)-f\left(x_{n}-f\left(x_{n}\right)\right)}, \\
& w_{n}=x_{n}+\gamma_{n} f\left(x_{n}\right), \quad n=1,2, \cdots, \\
& \lambda_{n}=-\frac{f\left(x_{n}+f\left(x_{n}\right)\right)-2 f\left(x_{n}\right)+f\left(x_{n}-f\left(x_{n}\right)\right)}{f\left(x_{n}\right)\left(f\left(x_{n}+f\left(x_{n}\right)\right)-f\left(x_{n}-f\left(x_{n}\right)\right)\right)}, \\
& y_{n}=x_{n}-\frac{f\left(x_{n}\right)}{\phi_{n}+\lambda_{n} f\left(w_{n}\right)}, \\
& x_{n+1}=y_{n}-\bar{\tau}_{n} \frac{f\left(y_{n}\right)}{\phi_{n}+\lambda_{n} f\left(w_{n}\right)}, \quad n=0,1, \cdots,
\end{aligned}
$$

where $\bar{\tau}_{n}$ is given by (17).

\section{RESULTS AND DISCUSSION}

We employed an iterative method (1) with (13), (17) and method (19) to solve nonlinear equations. We compared the new methods (1) and method (19) to X. Wang, T. Zhang [8] method (WZ), Dzunic [3] method ( $D z)$, Cordero et al. [1] method $(\mathrm{Co})$.

The numerical results are displayed in Tables 1-4. The comparison with existing methods is included as well. For numerical tests we considered the following problems:

$$
\begin{aligned}
& f_{1}=e^{x^{3}-x}-\cos \left(x^{2}-1\right)+x^{3}+1, \quad x^{*}=-1 \\
& f_{2}=e^{x^{3}-3 x} \sin x+\log \left(x^{2}+1\right), \quad x^{*}=0, \\
& f_{3}=M-E+\varepsilon \sin E, \quad 0<\varepsilon<1, x^{*}=0.38 \\
& f_{4}=e^{-x}+\frac{x}{5}-1, \quad x^{*}=4.96
\end{aligned}
$$

which has been selected from [5] and [8,9,10]. For $f_{1}, f_{2}$, we consider the initial guesses -0.6 and 1.3 , respectively. In particular, $f_{3}$ is Kepler's equation which relates the eccentric anomaly $E$, the mean anomaly $M$ and the eccentricity $\varepsilon$ in an elliptic orbit.

One of the classical laws of planetary motion due to Kepler says that a planet revolves around the sun in an elliptic orbit.

Suppose one needs to find the position $(x, y)$ of the planet at time $t$. This can be determined by the following formula:

$$
\begin{aligned}
& x=a \cos (E-\varepsilon) \\
& y=a \sqrt{1-\varepsilon^{2}} \sin E .
\end{aligned}
$$

To determine the position $(x, y)$, one must know how to compute $E$, which can be computed from Kepler's equation of motion:

$$
M-E+\varepsilon \sin E=0, \quad 0<\varepsilon<1 \text {. }
$$

The solution of the above equation can be obtained by implementing various iterative methods with different values of $M$ and $\varepsilon$. 
The approximate root of $f_{3}$ is 0.3899777749463621 .

In [10] we have considered one of the famous classical physics problem, which is known as the Planck's radiation law problem. Fourth non-linear function $f_{4}$ arises from this problem. Planck's radiation law problem calculates the energy density within an isothermal blackboard and is given by the following formula:

$$
\varphi(\lambda)=\frac{8 \pi c p \lambda^{-5}}{e^{\frac{c p}{\lambda \mathrm{BT}}-1}},
$$

where $\lambda$ is the wavelength of the radiation, $T$ is the absolute temperature of the blackbody, $B$ is the Boltzmann constant, $p$ is the Planck constant and $c$ is the speed of light. We are interested in determining wavelength $\lambda$ which corresponds to maximum energy density $\varphi(\lambda)$.

Further, $\varphi^{\prime}(\lambda)=0$. implies that the maximum value of $\varphi$ occurs when

$$
\frac{\frac{c p}{\lambda_{\mathrm{BT}}} e^{\frac{c p}{\lambda_{\mathrm{BT}}}}}{e^{\frac{c p}{\lambda_{\mathrm{BT}}}}}=5 .
$$

If $x=\frac{c p}{\lambda \mathrm{BT}}$, then the equation is satisfied when

$$
f_{4}=e^{-x}+\frac{x}{5}-1
$$

Table 1. Comparison of methods for example 1

\begin{tabular}{|c|c|c|c|c|}
\hline Methods & $\bar{\tau}_{n}$ & $n$ & $\left|x_{n}-x^{*}\right|$ & $\rho$ \\
\hline$(1),\left(\mathrm{p}=1, \lambda_{0}=-0.1, \gamma_{0}=-0.01\right)$ & $(17)$ & 3 & $0.37 \mathrm{e}-96$ & 6 \\
\hline 1$),\left(\mathrm{p}=2, \lambda_{0}=-0.1, \gamma_{0}=-0.01\right)$ & $(17)$ & 3 & $0.51 \mathrm{e}-168$ & 8 \\
\hline$(19),\left(\mathrm{p}=1, \lambda_{0}=-0.1, \gamma_{0}=-0.01\right)$ & $(17)$ & 3 & $0.69 \mathrm{e}-69$ & 6 \\
\hline$(19),\left(\mathrm{p}=2, \lambda_{0}=-0.1, \quad \gamma_{0}=-0.01\right)$ & $(17)$ & 3 & $0.71 \mathrm{e}-126$ & $\mathbf{8}$ \\
\hline WZ $[8],\left(\lambda_{0}=-0.1, \lambda_{n}=-H^{\prime \prime}{ }_{n} / 2 f^{\prime}\right)$ & - & 3 & $0.45 \mathrm{e}-98$ & 7 \\
\hline Dz [3], (p=-0.1, $\left.\gamma_{0}=-0.01, g=1+\theta_{n}\right)$ & - & 3 & $0.24 \mathrm{e}-168$ & 7 \\
\hline Co $[1],\left(\lambda_{0}=-0.1, \quad \gamma_{0}=-0.01\right)$ & - & 3 & $0.11 \mathrm{e}-167$ & 7 \\
\hline
\end{tabular}

Table 2. Comparison of methods for example 2

\begin{tabular}{|c|c|c|c|c|}
\hline Methods & $\bar{\tau}_{n}$ & $n$ & $\left|x_{n}-x^{*}\right|$ & $\rho$ \\
\hline$(1),\left(\mathrm{p}=1, \lambda_{0}=-0.1, \gamma_{0}=-0.01\right)$ & $(17)$ & 3 & $0.26 \mathrm{e}-94$ & 6 \\
\hline$(1),\left(\mathrm{p}=2, \lambda_{0}=-0.1, \gamma_{0}=-0.01\right)$ & $(17)$ & 3 & $0.79 \mathrm{e}-145$ & 8 \\
\hline$(19),\left(\mathrm{p}=1, \lambda_{0}=-0.1, \gamma_{0}=-0.01\right)$ & $(17)$ & 3 & $0.71 \mathrm{e}-91$ & 6 \\
\hline$(19),\left(\mathrm{p}=2, \lambda_{0}=-0.1, \gamma_{0}=-0.01\right)$ & $(17)$ & 3 & $0.88 \mathrm{e}-161$ & $\mathbf{8}$ \\
\hline $\mathrm{WZ}[8],\left(\lambda_{0}=-0.1, \lambda_{n}=-H^{\prime \prime}{ }_{n} / 2 f^{\prime}\right)$ & - & 4 & $0.40 \mathrm{e}-129$ & 7 \\
\hline $\mathrm{Dz}[3],\left(\mathrm{p}=-0.1, \gamma_{0}=-0.01, g=1+\theta_{n}\right)$ & - & 4 & $0.42 \mathrm{e}-360$ & 7 \\
\hline $\operatorname{Co}[1],\left(\lambda_{0}=-0.1, \gamma_{0}=-0.01\right)$ & - & 4 & $0.67 \mathrm{e}-245$ & 7 \\
\hline
\end{tabular}


Table 3. Comparison of methods for example 3

\begin{tabular}{|c|c|c|c|c|}
\hline Methods & $\bar{\tau}_{n}$ & $n$ & $\left|x_{n}-x^{*}\right|$ & $\rho$ \\
\hline$(1),\left(\mathrm{p}=1, \lambda_{0}=-0.1, \gamma_{0}=-0.01\right)$ & $(17)$ & 3 & $0.54 \mathrm{e}-105$ & 6 \\
\hline$(1),\left(\mathrm{p}=2, \lambda_{0}=-0.1, \gamma_{0}=-0.01\right)$ & $(17)$ & 3 & $0.61 \mathrm{e}-153$ & 8 \\
\hline$(19),\left(\mathrm{p}=1, \lambda_{0}=-0.1, \gamma_{0}=-0.01\right)$ & $(17)$ & 3 & $0.13 \mathrm{e}-102$ & 6 \\
\hline$(19),\left(\mathrm{p}=2, \lambda_{0}=-0.1, \gamma_{0}=-0.01\right)$ & $(17)$ & 3 & $0.68 \mathrm{e}-193$ & $\mathbf{8}$ \\
\hline $\mathrm{WZ}[8],\left(\lambda_{0}=-0.1, \lambda_{n}=-H_{n}^{\prime \prime} / 2 f^{\prime}\right)$ & - & 4 & $0.39 \mathrm{e}-133$ & 7 \\
\hline $\mathrm{Dz}[3],\left(\mathrm{p}=-0.1, \gamma_{0}=-0.01, g=1+\theta_{n}\right)$ & - & 4 & $0.52 \mathrm{e}-177$ & 7 \\
\hline $\operatorname{Co}[1],\left(\lambda_{0}=-0.1, \gamma_{0}=-0.01\right)$ & - & 4 & $0.17 \mathrm{e}-192$ & 7 \\
\hline
\end{tabular}

Table 4. Comparison of methods for example 4

\begin{tabular}{|c|c|c|c|c|}
\hline Methods & $\bar{\tau}_{n}$ & $n$ & $\left|x_{n}-x^{*}\right|$ & $\rho$ \\
\hline$(1),\left(\mathrm{p}=1, \lambda_{0}=-0.1, \gamma_{0}=-0.01\right)$ & $(17)$ & 3 & $0.18 \mathrm{e}-112$ & 6 \\
\hline$(1),\left(\mathrm{p}=2, \lambda_{0}=-0.1, \gamma_{0}=-0.01\right)$ & $(17)$ & 3 & $0.12 \mathrm{e}-172$ & 8 \\
\hline$(19),\left(\mathrm{p}=1, \lambda_{0}=-0.1, \gamma_{0}=-0.01\right)$ & $(17)$ & 3 & $0.26 \mathrm{e}-181$ & 6 \\
\hline$(19),\left(\mathrm{p}=2, \lambda_{0}=-0.1, \gamma_{0}=-0.01\right)$ & $(17)$ & 3 & $0.63 \mathrm{e}-198$ & $\mathbf{8}$ \\
\hline $\mathrm{WZ}[8],\left(\lambda_{0}=-0.1, \lambda_{n}=-H^{\prime \prime}{ }_{n} / 2 f^{\prime}\right)$ & - & 4 & $0.33 \mathrm{e}-162$ & 7 \\
\hline $\mathrm{Dz}[3],\left(\mathrm{p}=-0.1, \gamma_{0}=-0.01, g=1+\theta_{n}\right)$ & - & 4 & $0.32 \mathrm{e}-165$ & 7 \\
\hline $\operatorname{Co}[1],\left(\lambda_{0}=-0.1, \gamma_{0}=-0.01\right)$ & - & 4 & $0.33 \mathrm{e}-197$ & 7 \\
\hline
\end{tabular}

\section{CONCLUSIONS}

In this paper, a new two-point derivativefree iterative method with memory for solving nonlinear equations was introduced and studied. Using optimal values of parameters, the higher order method with memory is obtained. Exact analytical formulas for the optimal values of the parameters have been found for the first time. The convergence order increased from four to eight without any additional computations. Finally, numerical experiments have shown that the new method is

\section{REFERENCES}

1. A. Cordero, T. Lotfi, P. Bakhtiari, J. R. Torregrosa, An efficient twoparametric family with memory for nonlinear equations, Numer. Algor. 2015, 68: pp.323-335.

2. I. K. Argyros, M. Kansal, V. Kanwar, S. Bajaj, Higher-order derivative-free families of Chebyshev-Halley type methods with or without memory for solving nonlinear equations, Appl. Math. Comput. 2017, 315: p.224-245.

3. J. Dzunic On efficient two-parameter methods for solving nonlinear eight-order and effective. We also solved some real world applications of different nature to show the effectiveness of the proposed methods.

Acknowledgments: The authors wish to thank the anonymous referees for their valuable suggestions and comments, which improved paper.

The work was supported partially by the Mongolian Foundation for Science and Technology under Grant SST\$\\$18/2018. equations, Numer. Algor. 2013, 63: 549-569.

4. M. S. Petkovic, S. Ilic, J. Dzunic Derivative-free two-point methods with and without memory for solving nonlinear equations, Appl. Math. Comput. 2010, 217:pp.1887-1895.

5. T. Lotfi, F. Soleymani, M. Ghorbanzadeh, P. Assari, On the construction of some tri-parametric iterative methods with memory, Numer. Algor. 2015, 70:pp.835-845.

6. T. Zhanlav, Kh. Otgondorj, On the optimal choices of parameters in the 
two-point iterative methods for solving nonlinear equations, Comput. Math. Math. Phys. 2021, 61: pp.29-42

7. T. Zhanlav, O. Chuluunbaatar, Kh. Otgondorj, A derivative-free families of optimal two-and three-point iterative methods for solving nonlinear equations, Comput. Math. Math. Phys. 2019, 59: pp.920-936.

8. X. Wang, T. Zhang, A new family of Newton-type iterative methods with and without memory for solving nonlinear equations, Calcolo 2014, 51: pp.1-15.

9. X. Wang, A new accelerating technique applied to a variant of Cordero-Torregrosa method, J. Comput. Appl. Math. 2018, 330: pp.695-709.

10. I. K. Argyros, M. Kansal, V. Kanwar, S. Bajaj, Higher order derivative-free families of Chebyshev-Halley type methods with or without memory for solving nonlinear equations, Appl. Math. Comput. 2017,315: p.224-245. 